\title{
Design and Experiments with a 30 DOF Robot
}

\author{
Gregory S. Chirikjian ${ }^{1}$ \\ Dept. of Mechanical Engineering \\ Johns Hopkins University \\ Joel W. Burdick \\ Dept. of Mechanical Engineering \\ California Institute of Technology
}

\begin{abstract}
In previous work, the authors developed a novel framework for 'hyper-redundant' robot kinematic analysis and algorithm development. In order to validate and demonstrate that work, a 30 degree-of-freedom planar hyper-redundant manipulator was constructed. This paper reviews the implementation of this robot and summarizes experimental work in hyper-redundant manipulation, obstacle avoidance, and locomotion. Potential benefits of high-dof manipulators include superior obstacle avoidance ability, increased robustness with respect to mechanical failure, and the ability to perform new forms of robot locomotion and grasping.
\end{abstract}

\section{Introduction}

"Hyper-redundant" manipulators are redundant manipulators with a very large or infinite degree of redundancy. These manipulators can be analogous in morphology and operation to "snakes," "elephant trunks," or "tentacles." Because of their highly articulated structures, these robots are potentially superior for operations in highly constrained and unusual environments. Hyper-redundancy can also be used to implement novel forms of robotic locomotion analogous to the motion of worms, slugs, and snakes. Further, hyper-redundant robots can have increased robustness with respect to mechanical failure.

To date, hyper-redundant manipulators have remained largely a laboratory curiosity. There are a number of reasons for this: (1) previous kinematic modeling techniques have not been particularly efficient or well suited to the needs of hyper-redundant

\footnotetext{
${ }^{1}$ This work was performed while the first author was a graduate student at Caltech
}

robot task modeling; (2) the mechanical design and implementation of hyper-redundant robots has been perceived as unnecessarily complex; and (3) hyperredundant robots are not anthropomorphic, and pose difficult programming problems. The authors have undertaken a broadly based investigation which we hope will eventually overcome many of the obstacles to practical hyper-redundant robot applications.

In previous and concurrent papers, the authors have presented novel techniques for efficient kinematic analysis of hyper-redundant robots [2, and references therein]. These techniques have also been used as the basis for novel obstacle avoidance, locomotion, and grasping schemes $[2,3]$. These works should be consulted for references to other approaches to hyperredundant mechanism analysis.

This paper presents the design and implementation of a thirty degree-of-freedom planar hyper-redundant manipulator. This manipulator was developed to demonstrate the practicality of hyper-redundant manipulators for a variety of tasks. Experiments in hyperredundant obstacle avoidance, grasping, object manipulation, and dual hyper-redundant arm grasping are presented.

Hyper-redundant robots have previously been called 'highly articulated,' 'tentacle,' 'snake-like,' 'tensorarm,' 'elephant-like,' and 'spine.' The authors have coined the word 'hyper-redundant' to capture the essence of these related concepts. To our knowledge, the earliest hyper-redundant robot designs/implementations date to the late 1960's [1]. Hirose and coworkers [4] have implemented a large number of working high-dof systems, and have recently adapted the term 'hyper-redundant' [5]. Numerous other authors have suggested hyper-redundant designs or developed hyper-redundant robot mechanisms. Examples include $[6,8]$. Many of these designs were driven 
to some extent by a particular application or operating environment scenario.

In this paper we present a design which is not focused on a particular application, but which is most suited for demonstrating the widest variety of hyperredundant robot operating modes. Some of these operating modes, such as grasped object reorientation, have not previously been considered or demonstrated in the literature.

Section 2 overviews different hyper-redundant manipulator morphologies, and includes some comments about the relative merits of each type. Section 3 describes the functional requirements for our system and details the design and implementation of our hyperredundant robot. Section 3 also includes some philosophical arguments in favor of hyper-redundant robot systems for some application domains. Section 4 reviews experiments performed with this system.

\section{Candidate Morphologies}

By a morphology, we mean the choice and arrangement of a hyper-redundant robot's actuators and mechanical structure. The selection of a particular morphology will obviously depend heavily upon the functional and operational requirements of a particular application. Some possible morphologies are represented in Figure 1. To obtain the maximum possible benefit from their highly articulated structures, hyper-redundant manipulators must not only be able to place their endeffectors at a specified location, but must also be able to:

- maneuver through highly constrained workspaces.

- grasp objects in a tentacle-like fashion

- generate peristaltic waves to manipulate objects

These functional requirements arise in the following anticipated application scenarios:

- operations in highly constrained environments, such as: nuclear reactor steam generator maintenance; chemical sampling in buried toxic waste containers; and medical endoscopy.

- tentacle-like grasping and object reorientation such as: capturing and despinning free floating satellites; replacement of mechanical components in hard to reach areas of an automobile; and complex 'whole arm' manipulation experiments.
- exploration in complex environments such as: emergency response vehicles in burning or collapsed buildings; and sensor placement in complicated geological formations, such as lava tubes.

Naturally, no single design can be suitable for all applications. Rather than discuss the morphologyapplication relation, we will focus on the enumeration of broad design classes and their strengths and weaknesses. Section 3 reviews our actual implementation in the context of this discussion.

Hyper-redundant manipulators can be physically implemented using a variety of actuators and linkages, such as pneumatic bellows [8], rubber gas actuator driven devices [6], a serial chain consisting of a large number of rigid links [2], or a variable geometry truss $[2,7]$. These morphologies can be roughly categorized into three main types: (1) discrete morphologies (Figure 1(a)) which have a large, but finite, number of rigid links; (2) continuous morphologies (Figure 1(b)) in which the robot actuation is distributed over the manipulator length and is continuously deformable; and (3) cascades of parallel platform modules such as variable geometry trusses (VGTs)(Figure 1(c)) or complex linkages (Figure 1(d)). For the most part, the distinction in morphologies arises from the types of actuators chosen, and their means of deployment in the robot's structure.

In continuous morphologies, actuation is distributed through the robot structure, resulting in the ability to continuously deform the robot's local geometry. Continuous morphologies include robots based on flexible pneumatic and hydraulic actuators and actuator bundles. Continuous manipulators have the most general geometry, and at first glance would appear to be more maneuverable and highly articulated. However, their actual implementation can be problematical. Truly distributed actuation (akin to muscles in a snake) is difficult to engineer in practice. Further, pneumatic and hydraulic schemes are not very suitable for locomoting hyper-redundant robots, as it is difficult to carry the pumps, regulators, and other required components in a self-propelled package.

Discrete morphologies are comprised of a finite number of non-distributed actuators. Examples of such systems include serial chain rigid link robots (with revolute and/or prismatic joints),variable geometry truss manipulators, or any of a variety of in-series platform manipulator modules [2]. Serial chain rigid link systems are an extension of traditional manipulator design methodology, have a simple kinematic structure, and have simple 'fitting' algorithms [2]. However, this 
morphology has inherently poor mechanical advantage, making it difficult to build one which can support its own weight when fully extended. In addition, serial chain designs are not robust with respect to actuator failure.

Variable geometry truss systems have many advantages for practical hyper-redundant manipulator implementation. VGTs were originally studied for application to large space-structures, such as precision segmented mirrors. Examples in the literature are numerous $[7,9]$. More recently, variable geometry truss manipulators (VGTMs) have been proposed and investigated $[10,11]$. Traditional VGTs and VGTMs can be differentiated by their actuation requirements. For large space structures, the primary role of the actuators and control system is to control structural resonances. Thus, these systems have actuators with high bandwidth and very little stroke. Conversely, VGTs for robotics applications require actuators with substantial stroke to effect significant changes in the structure geometry. We have chosen a VGT geometry for our system because of its inherent mechanical advantage which is required for several of the experiments in hyper-redundant grasping. Section 3 will elaborate on this design, and other relative merits of of VGT systems.

Most actuator technologies and implementations fall clearly into one category or the other. However, some actuators, such as tendons or shape memory alloys, can be deployed in either a continuous or discrete fashion. For example, tendons can be distributed throughout a hyper-redundant structure to provide continuous or discrete changes in geometry, depending upon the way they are attached to the robot structure. Tendons can either be active, e.g. constructed from contracting shape memory alloys or contracting polymers. Or, they can be passive, and are actuated by pulling (typically with a d.c. servo motor). A number of tendon based structures have been suggested and constructed [1]. Tendon based structures can be very light in weight. However, active tendons based on SMAs are typically slow in response. Passive tendons require remotely mounted actuators, and tendon routing becomes very complicated and impractical for the large numbers of tendons required to implement versatile spatial hyper-redundant robots. Further, nearly all tendon based designs implemented to date do not have complete kinematic functionality. That is, these designs can not provide twisting motion about the manipulator's 'backbone', nor do they have a general capability to implement broad changes in the torsion and curvature of the backbone. Consequently, they can only assume a restricted class of shapes.

In the following section, we outline the functional requirements for our system, and the subsequent design which best met these requirements.

\section{Manipulator Description}

For simplicity's sake, we have chosen to focus on the development of a planar hyper-redundant robot, leaving the development of a spatial system for future work. The primary functions of this robot are to demonstrate and validate our previous analytical work, and to serve as the basis for future work in hyperredundant robot analysis, design, programming, and sensor-based operation. Consequently, such a robot has the following functional requirements:

- a large workspace for end-effector placement.

- the ability to demonstrate obstacle avoidance in highly constrained spaces.

- the ability to grasp objects in a tentacle-like fashion, and manipulate these objects according to the 'grasping wave' schemes outlined in $[2,3]$.

- the ability to disassemble the robot in to two separate, but identical, hyper-redundant halves for dual arm grasping and object manipulation.

Our final design is a 30 degree-of-freedom VGT structure consisting of 10 identical three degree-offreedom truss modules, or 'bays.' Each truss module (Figure 2) contains 3 prismatic joints. In effect, each bay is a planar parallel manipulator. The prismatic actuators are actually implemented with D.C. servo motors and lead screw drives. The actuators can vary in length from a minimum of 12 inches to a maximum of 18 inches, and can generate a force of 75 points during motion, and can withstand 225 pounds staticly. Each actuator is equipped with a linear potentiometer to measure its absolute displacement. The accuracy of the feedback potentiometer, and the backlash in the lead screw are both approximately 1 percent of maximum extension.

For manipulation and grasping experiments, castors are attached to the fixed elements of the modules in order to allow low friction motion over the laboratory floor, which is the plane of manipulation. Detachable foam 'fingers' are used as the contact points for planar grasping operations. Because of its modular shape, 
the robot can be easily separated into two 15 degree-offreedom manipulators for dual arm manipulation experiments.

Figure 2 shows one module, or bay, of the truss robot. Let the length of a module (from fixed element to fixed element as measured in a reference configuration) divided by the width of the module (distance between actuator pivot points) be called the module aspect ratio. The fixed elements of the truss modules are designed so that each module's aspect ratio can be varied for different applications. A very high module aspect ratio leads to greater dexterity, but has reduced mechanical advantage. High aspect ratios are advantageous for obstacle avoidance in highly constrained spaces. Conversely, low aspect ratios provide greater mechanical strength at the expense of dexterity. For most applications, the module aspect ratios are uniformly sized, though they can be non-uniform when necessary. For example, in manipulation, the modules closest to the base are subjected to greater forces and moments. Their aspect ratios can be reduced to improve their strength.

The robot system is controlled by an a multiprocessing computer, which consists of Heurikon 68030 processing boards in a VME bus, which is in turn connected to a Sun Microsystems 4/260 computer. The WindRiver Systems VxWorks software system provides operating system and system management functions. The 68030 processors compute the kinematic, path planning, sensor processing, and task level coordination functions. Low level control of the actuator positions is also performed by 68030 processors. The Sun computer is used primarily for user interface and data storage.

As demonstrated by our design, hyper-redundant robots do have some inherent design and operating advantages.

First, hyper-redundant robot design is often amenable to simple modular implementation. In this planar case, each module consists of a planar parallel manipulator. For spatial manipulators, the module geometry can be a Stewart platform. Simple mechanical adjustments can alter the mechanical advantage properties of the modules to suit different needs.

Second, in most operational scenarios, only a small fraction of the robot actuators are actually in use, minimizing their duty cycle and prolonging their life. Even so, such systems are highly fault tolerant with respect to Intmerous actuator failures. For instance, in this planar thirty degree of freedom manipulator, ninety percent of the actuators can fail, and the system will retain the ability to position and orient objects at the end-effector (though obstacle avoidance capability and the workspace volume would be severely reduced).

Third, assembly and maintenance of this hyperredundant manipulator is extremely simple. In particular, the entire mechanism can be assembled from its constituent parts in less than one half hour (with 3 people working in parallel). Partial disassembly to replace actuators is trivial. The pins which fix the relative position of each actuator in the overall structure can be removed, and an actuator can be replaced in less than 5 minutes. For dual-arm experiments, the 10-bay truss can be 'split' into two 5-bay units (which are still hyper-redundant) in a matter of minutes also.

Such systems can also be weight efficient. The 30 degree-of-freedom robot can contract to a minimum length of 12 feet and extend to a maximum length of 18 feet. Yet the robot weighs less than $55 \mathrm{~kg}$.

Of course, hyper-redundant robots have obvious disadvantages, and are best suited to niche applications. Small errors in each actuator position can accumulate into large base-to-tip position errors. Thus, hyperredundant manipulators are at a disadvantage for precise positioning tasks, unless they are instrumented with numerous sensors and equipped with sensor-based control strategies which adjust for local errors. This is a subject for future research.

\section{Experiments}

This section describes the task scenarios which are being tested with the 30 degree-of-freedom variable geometry truss which is the focus of this paper.

Obstacle Avoidance : The theory developed in [2] for obstacle avoidance is implemented in Figure 3. Here the manipulator is demonstrating a task which would be insurmountable by standard kinematically sufficient manipulators.

Single-Arm Grasping : The photos in Figure 4 show how a hyper-redundant manipulator can be used as a grasping device. In this particular picture, a single hyper-redundant manipulator is grabbing a mock satellite, to be recovered from orbit.

Dual Arm Manipulation : A variant on the single arm grasping and manipulation scheme is shown in Figure 5. In this case, two hyper-redundant arms work together, as formulated in [2]. 


\section{Summary}

This paper presented the design and implementation of a 30 degree-of-freedom planar hyper-redundant robot. This system was developed to validate the authors' previous analytical investigations of hyper-redundant robot kinematics and redundancy resolution. In previous work, we developed techniques which were highly efficient and sufficiently general to enable real-time inverse kinematics and motion planning for this complex class of robots. Further, this basic framework has been used to develop novel obstacle avoidance, locomotion, and grasping algorithms. The experiments detailed in this paper confirm the validity of our previous work. Further, our experience in constructing this system shows that hyper-redundant manipulator design need not be overly complex. Our hope is that our analytical and experimental investigations of hyper-redundancy will bring the concept of hyper-redundancy closer a level of practical real-time implementation.

\section{Aknowledgements}

This work was sponsored in part by a NASA Graduate Student Researchers Program fellowship (for the first author), the Caltech President's Fund, \# PF-331, and the National Science Foundation, grant MSS-901779.

\section{References}

[1] Anderson, V.V., Horn, R.C., "Tensor-arm Manipulator design," ASME Trans., Vol. 67-DE-57, pp. 1-12, 1967.

[2] Chirikjian, G.S., "Theory and Applications of Hyper-Redundant Robotic Manipulators," Ph.D Dissertation, Department of Applied Mechanics, Division of Engineering and Applied Science, California Institute of Technology, June 1992.

[3] Chirikjian, G.S., Burdick, J.W., "Kinematics of Hyper-Redundant Locomotion with Applications to Grasping," 1991 IEEE Conference on Robotics and Automation, April, 1991.

[4] Hirose, S., Umetani, Y., "Kinematic Control of Active Cord Mechanism With Tactile Sensors," Proceedings of Second International CISM-IFT Symposium on Theory and Practice of Robots and Manipulators, pp. 241-252, 1976.
[5] [15] Hirose, S., Yokoshima, K., Ma, S., "2 DOF Moray Drive for Hyper Redundant Manipulator," Proceedings, IROS'92, pp. 1735-1740B, Raleigh, NC, July 7-10, 1992.

[6] Suzumori, K., Iikura, S., Tanaka, H., "Development of Flexible Microactuator and It's Applications to Robotic Mechanisms," 1991 IEEE Conference on Robotics and Automation, Sacramento, CA, April, 1991.

[7] Wada, B., "Adaptive Structures : An Overview," J. Spacecraft, Vol. 27., No. 3., May-June 1990.

[8] Wilson, J.F., Mahajan, U., "The Mechanics and Positioning of Highly Flexible Manipulator Limbs," Journal of Mechanisms, Transmissions, and Automation in Design. Vol, 111, June 1989.

[9] Miura, K., Furuya, H., "Variable Geometry Truss and Its Application to Deployable Truss and Space Crane Arm," Acta Astronautica, Vol. 12, No. $7 / 8,1985$, pp. 599-607.

[10] Naccarato, F., Hughes, P.C., "Inverse Kinematics of Variable-Geometry Truss Manipulators," Journal of Robotic Systems Vol. 8, No. 2, pp. 249-266, 1991.

[11] Salerno, R.J., Reinholtz, C.F., Robertshaw, H.H., "Shape Control of High Degree-of-Freedom Variable Geometry Trusses," Proceedings of the Workshop on Computational Aspects in the Control of Flexible Systems, Part 2, Williamsburg, VA, July 12-14, 1988. 

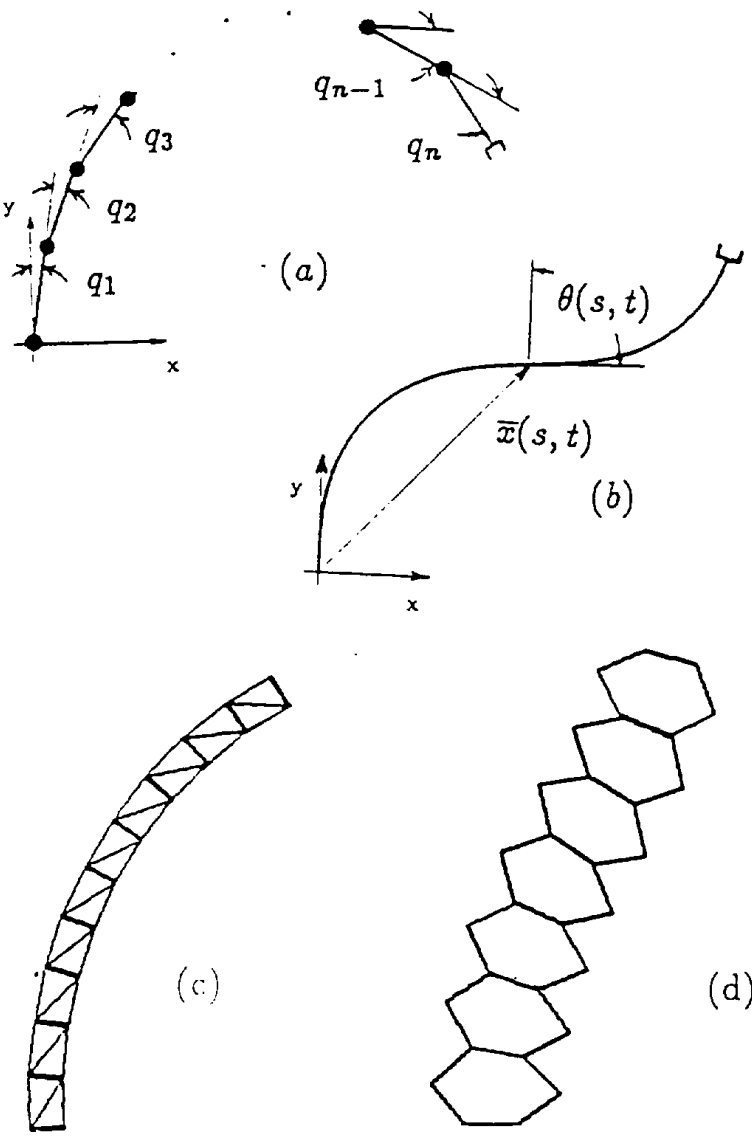

Figure 1: Hyper-Redundant Morphologies

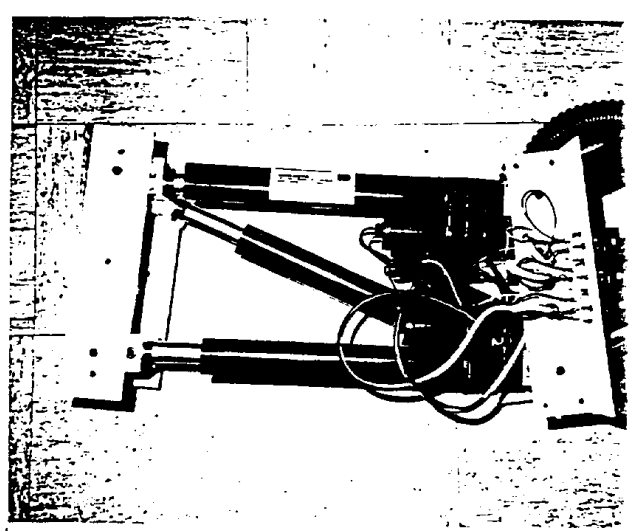

Figure 2: One Truss Module
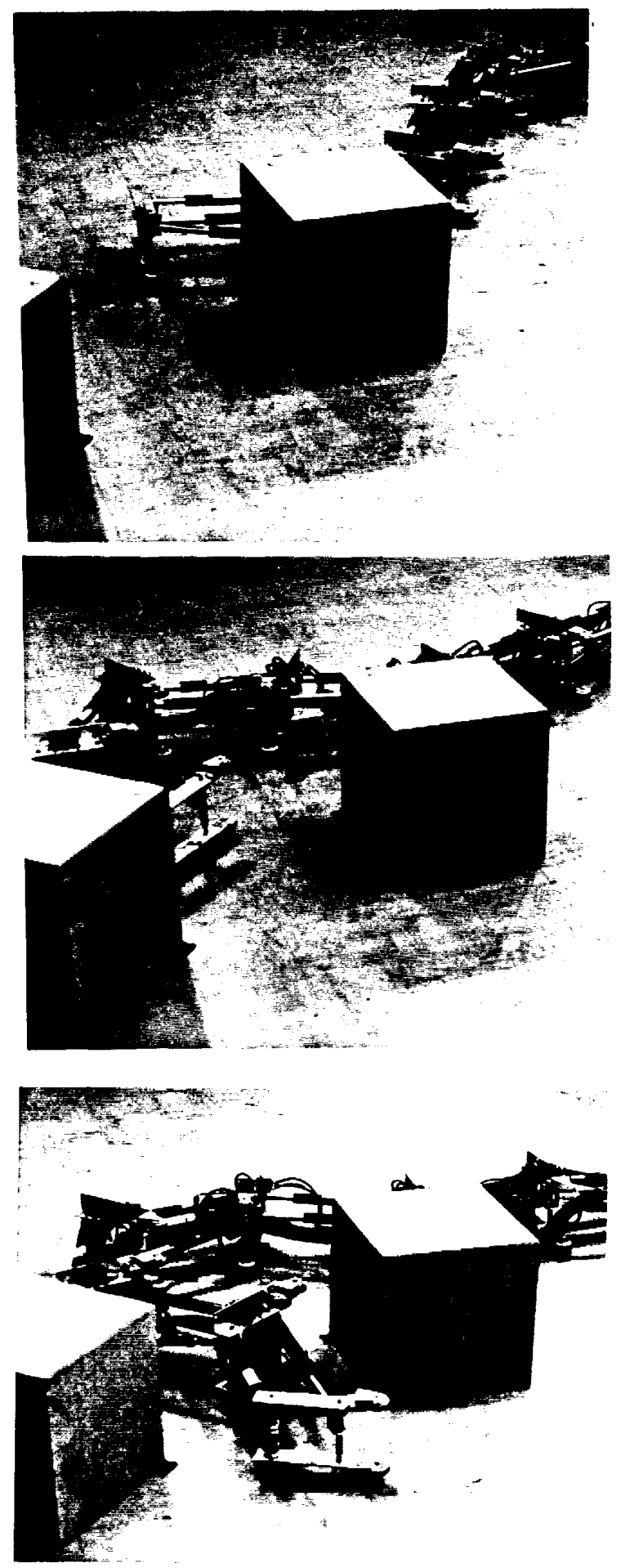

Figure 3: Obstacle Avoidance 


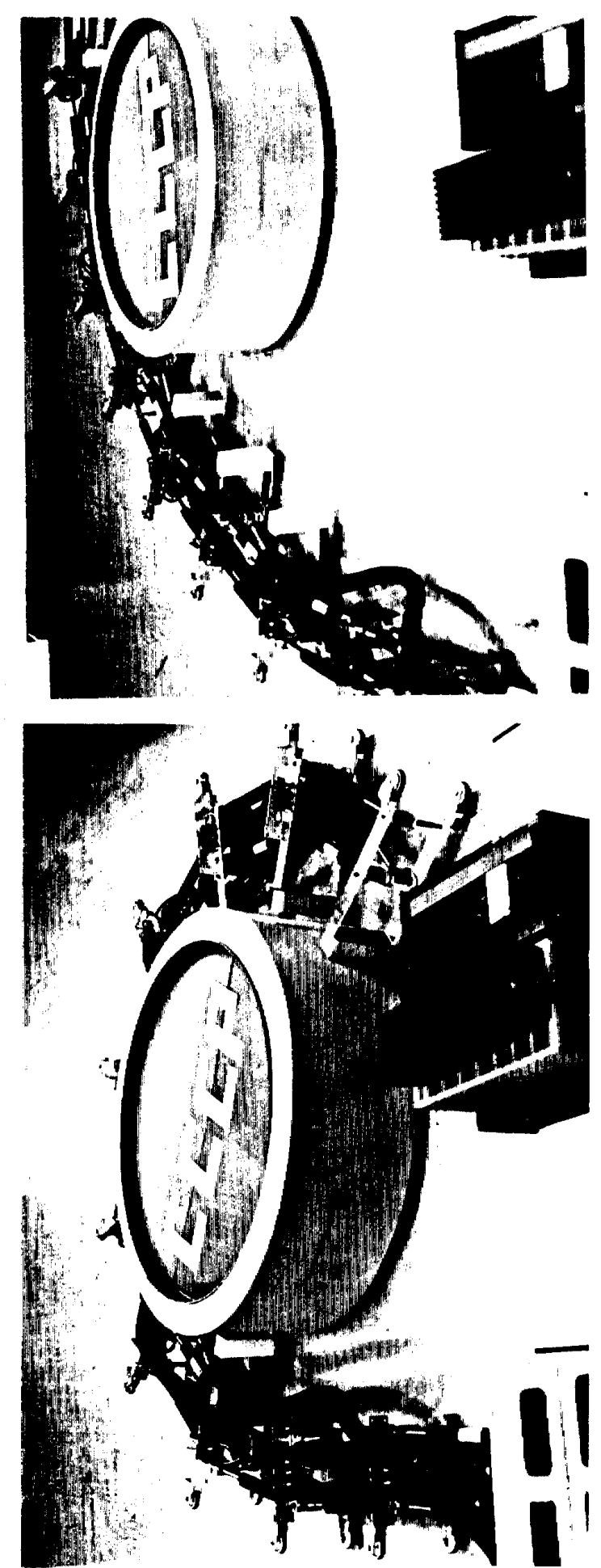

Figure 4: Single-Arm Satellite Recovery
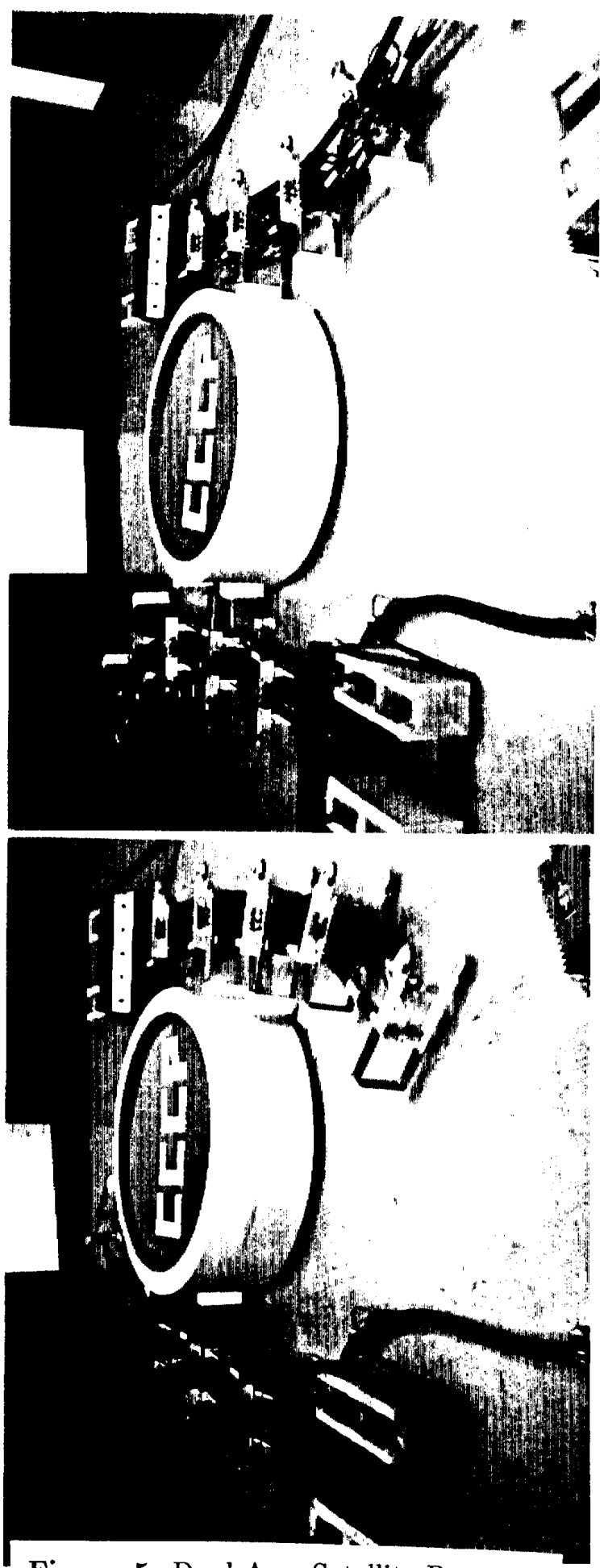

Figure 5: Dual-Arm Satellite Recovery 